\title{
Treatment options for obstructive sleep apnea
}

Patrick Pavwoski, DO; Anita Valanju Shelgikar, MD

\begin{abstract}
Purpose of review: Obstructive sleep apnea (OSA) is a global problem with implications for general health and quality of life, and is often encountered in patients with neurologic disease. This review outlines treatment modalities to consider for management of OSA in patients with neurologic disease. Recent findings: New advances in positive airway pressure (PAP) devices, oral appliances, and surgical interventions offer a wide range of treatment options for patients with OSA. Summary: PAP therapy remains the gold standard treatment for OSA. Other treatment modalities may be considered for OSA patients who decline or cannot tolerate PAP therapy. Some OSA patients may benefit from multimodal treatment. Neurol Clin Pract 2017;7:77-85
\end{abstract}

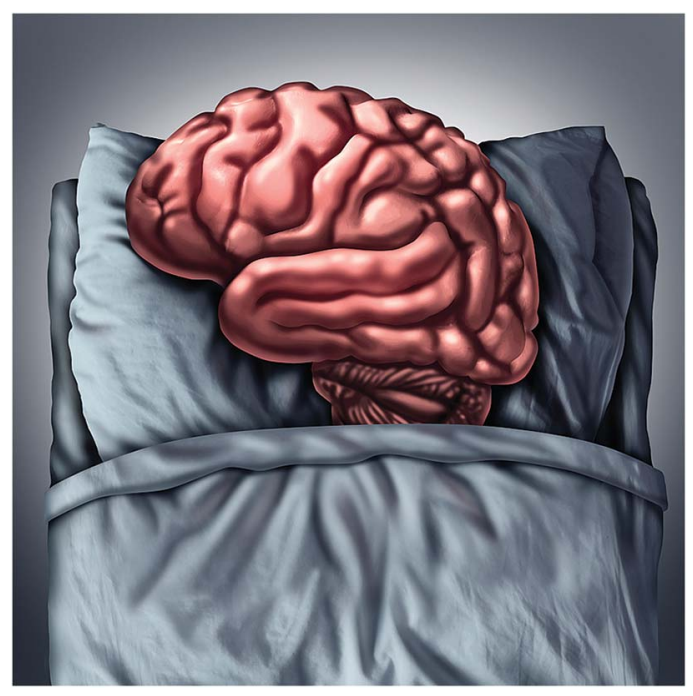

$\mathrm{P}$ atients with multiple sclerosis, epilepsy, neurodegenerative disease, and other categories of neurologic disease often look to the treating neurologist for evaluation and management of fatigue and tiredness. While fatigue may be a component of the underlying neurologic condition or medication side effect, it may also reflect comorbid obstructive sleep apnea (OSA). This brings recognition and treatment of OSA into the arena of the general neurologist, who often seeks to improve patients' daytime functioning without the addition of multiple medications. Having a working knowledge of OSA and available treatment options allows general neurologists to counsel patients about how identification and appropriate treatment of OSA may help improve daytime fatigue and reduce risk of comorbidities associated with untreated OSA.

OSA affects $9 \%-26 \%$ of the middle-aged population ${ }^{1}$ and is characterized by $\geq 15$ obstructive events per hour of sleep or $\geq 5$ obstructive events per hour of sleep and the presence of any related symptoms (snoring, witnessed apnea, nocturnal gasping or choking, nonrestorative sleep, excessive daytime sleepiness). ${ }^{2}$ OSA incidence in patients with certain neurologic conditions, such as stroke or medication-refractory epilepsy, is higher than in the general population. ${ }^{3,4}$ Untreated OSA can lead to daytime sleepiness, decreased productivity, increased motor vehicle accidents, and worsening hypertension, atrial fibrillation, and stroke., Appropriate evaluation and treatment of OSA may yield symptomatic benefit for patients with comorbid neurologic disease.

Garden City Hospital (PP); and University of Michigan (AVS), Ann Arbor.

Funding information and disclosures are provided at the end of the article. Full disclosure form information provided by the authors is available with the full text of this article at Neurology.org/cp.

Correspondence to: pavwosk2@gmail.com 


\section{CPAP is considered the gold standard} treatment for OSA because its use can improve sleep-related symptoms and quality of life.

The table summarizes the OSA treatment options that are discussed in detail.

\section{What treatment should I consider first for my OSA patients?}

Continuous positive airway pressure (CPAP) CPAP is considered the gold standard treatment for $\mathrm{OSA}^{7}$ because its use can improve sleep-related symptoms and quality of life. CPAP acts as a pneumatic splint that stabilizes the upper airway with constant positive pressure via a mask interface. CPAP has been shown to significantly decrease the apnea-hypopnea index (AHI), an average that represents the combined number of apneas and hypopneas that occur per hour of sleep, particularly in patients with severe disease (defined as an AHI $\geq 30 / \mathrm{h}$ ). ${ }^{1}$ $\mathrm{CPAP}$ users also show improvements in objective and subjective sleepiness compared to controls. $^{8}$

Despite these benefits, nightly CPAP use remains problematic for some patients. ${ }^{9}$ Difficulty with CPAP may stem from mask discomfort or claustrophobia, pressure intolerance, lifestyle or social considerations, or a combination of these factors.

CPAP: Consider this gold standard treatment modality as first-line for all OSA patients.

\section{What if my OSA patients have trouble tolerating the CPAP setting?}

Individuals with pressure intolerance may experience dryness or irritation of nasal and pharyngeal membranes, nasal congestion, or eye irritation from air leakage with CPAP use. Use of heated humidification ${ }^{10}$ or different mask types may facilitate comfort and increased CPAP use. Short-term reduction of the pressure setting ${ }^{10}$ may help the patient become acclimated to

Table Treatment options for obstructive sleep apnea

Positive airway pressure (PAP)

Continuous PAP (CPAP)

Bi-level PAP

+/- Backup rate

+/- Average volume assured pressure support

Autotitrating PAP (may be CPAP or bi-level PAP)

Oral appliances

Tongue-retaining devices

Mandibular advancement devices

Surgical

Phase I (nasal, palatal, tongue)

Phase II (maxillomandibular advancement)

Hypoglossal nerve stimulation

Adjunct

Weight loss

Positional therapy

Nasal expiratory PAP

Noninvasive oral pressure therapy 
$\mathrm{CPAP}$, with the ultimate goal to eventually resume the prescribed pressure. Flexible pressure at end-expiration may also increase patient comfort with the pressure.

Other influences on adherence include apprehension about how CPAP will affect appearance or interfere with sexual functioning, the patient's and bed partner's perceived benefit, if the patient sleeps alone, socioeconomic status, and anxiety or depression. ${ }^{10}$ Open discussions can help mitigate negative perceptions of CPAP therapy and work towards nightly CPAP use.

Bi-level positive airway pressure (PAP) Bi-level PAP was originally developed to administer varying pressures between the inspiratory and expiratory cycle, one pressure for inhalation and the second for exhalation. This may facilitate tolerance of higher pressures via easier exhalation. During the inspiratory cycle, the greater level of pressure combats the inspiratory flow limitation in the upper airway. ${ }^{11}$ This yields a greater tidal volume and unloading of the respiratory muscles compared to CPAP and essentially treats nonobstructive sleep-related hypoventilation. ${ }^{11}$ Some bi-level PAP devices have additional capabilities, including backup breath delivery in a spontaneous or timed mode.

Bi-level PAP with average volume-assured pressure support adapts pressure support to guarantee a predetermined tidal volume. ${ }^{12}$ This noninvasive ventilation modality may be considered for patients with obesity hypoventilation, chronic obstructive pulmonary disorder, or alveolar hypoventilation seen in neuromuscular disorders such as amyotrophic lateral sclerosis (ALS), spinal muscular atrophy, myotonic dystrophy, or cervical spinal cord injury. ${ }^{12,13}$ In one study comparing patients with little or no bulbar involvement ALS, the use of a noninvasive ventilator had been shown to increase median survival in the noninvasive ventilation group for 48 days longer for a total of 219 days vs 171 days in the standard care group. ${ }^{14}$

Bi-level PAP: Consider for OSA patients with intolerance to CPAP or those who have hypoventilation in the setting of obesity or neuromuscular disease.

Autotitrating PAP (APAP) APAP is set to a pressure range and the delivered pressure varies depending upon a device-specific algorithm. Both CPAP and bi-level PAP devices are available in autotitrating modes. Generally the device defines normal airflow for the patient, and delivers additional pressure when the flow curve is restricted. ${ }^{15}$ APAP devices may be useful for patients whose titration-recommended setting was not suitable due to the inherent limited sampling of a single night or half-night titration study. ${ }^{11,15}$ APAP therapy may also be considered for patients in whom large weight fluctuations ${ }^{11}$ are anticipated, such as pregnant patients or those undergoing bariatric surgery. Contraindications to APAP include congestive heart failure, lung disease such as chronic obstructive pulmonary disease, obesity hypoventilation syndrome, lack of snoring, or central sleep apnea.

APAP: Can consider for OSA patients who do not have clear contraindications to APAP use.

\section{Can my OSA patients use an oral appliance for treatment?}

Oral appliance therapy for OSA Oral appliances can benefit carefully selected OSA patients who prefer not to use CPAP or have comprehensive surgeries. Oral appliances improve upper airway patency during sleep by enlarging the upper airway or by decreasing upper airway collapsibility. ${ }^{16}$ Oral appliances can be categorized as tongue retaining devices and mandibular advancement devices (MADs), with the latter being more commonly used. MADs cover the upper and lower teeth and hold the mandible in a forward position with respect to the resting position and can be customizable or over-the-counter. ${ }^{16}$ Mandibular protrusion advances the tongue position and subsequently increases oropharyngeal volume. ${ }^{17}$

\section{Is my OSA patient a candidate for a MAD?}

$\mathrm{MADs}$ are indicated for patients with mild to moderate OSA or those with severe OSA who are unwilling or unable to use CPAP, have good dentition, and have a body mass index (BMI) below $30 \mathrm{~kg} / \mathrm{m}^{2}{ }^{2}$ A dentist trained in dental sleep medicine examines the mandible, bite, dentition, and range of motion to identify patients with temporomandibular joint 
dysfunction ${ }^{6}$ that may worsen with MAD use. Accredited sleep laboratories may also offer polysomnographic evaluation with a remotely controlled mandibular positioner to help predict an effective mandibular protrusion position. This technology is currently available for clinical use at sleep laboratories in many geographic regions. ${ }^{18}$

In patients with mild to moderate OSA, a $>50 \%$ reduction in AHI to $<5 / \mathrm{h}$ occurred in $42.8 \%$ of patients using an oral appliance and $73.2 \%$ of patients using CPAP. ${ }^{19,20}$ Odds of achieving AHI $<5 / \mathrm{h}$ and $<10 / \mathrm{h}$ was respectively 49 times greater and 89 times greater with the oral appliance-treated group compared to the control untreated group. ${ }^{19,20}$ Compared to noncustom MADs, custom MADs have been shown to achieve greater reduction in AHI. ${ }^{19}$

Adherence to oral appliance therapy is difficult to track objectively and thus compare directly with rates of CPAP adherence. Thermosensors can be embedded into oral appliances to monitor device usage, ${ }^{21}$ but presence of these sensors is not ubiquitous. Overall, the absolute difference between the mean subjective adherence rates for oral appliance users was 0.70 more hours per night than the objective adherence rates among CPAP users. ${ }^{19,20}$

MADs: Consider for patients with mild to moderate OSA, or those with severe OSA who have chosen not to use or are unable to use CPAP.

\section{What surgical treatment options are available to my OSA patients?}

Because OSA relates to the structure and tone of the upper airway, upper airways surgeries are aimed at reducing the degree of obstruction in the nose, oropharynx, or hypopharynx. Anatomical features that may predispose to OSA include retroposition of the maxilla or mandible, enlarged pharyngeal fat pads, enlarged soft palate, enlarged tongue, narrow posterior airway space, and upper airway hypotonia.

Phase I surgeries include nasal surgeries, palatal surgeries, and tongue-based surgeries that may initially alleviate and treat OSA. Phase II surgery, known as maxillomandibular advancement, is generally reserved for patients with major OSA due to velo-orohypopharyngeal airway obstruction that could not be resolved from phase I surgeries. ${ }^{22-24}$

Surgical success is frequently defined when the individual achieves a greater than $50 \%$ reduction of the AHI or an AHI of $\leq 20 / h^{25,26}$ However, this oft-used definition of success for OSA surgeries may not represent a clinical "cure," so careful attention must be paid to endpoints used in studies of surgical treatments for OSA. ${ }^{27}$ Determination of surgical candidacy on a case-by-case basis, with attention to severity of OSA, presence/absence of obesity, and presence/absence of craniofacial features amenable to surgery, can help increase the likelihood of surgical success. Continuous weight management and optimal treatment of medical comorbidities is imperative to reduce postoperative worsening or recurrence of OSA.

\section{What are the initial (phase I) surgeries to consider for my OSA patients?}

In general, phase I surgeries should be considered for patients with mild to moderate OSA who are nonobese.

Adenotonsillectomy Adenotonsillar hypertrophy has become the most common cause of pediatric OSA, ${ }^{28}$ for which adenotonsillectomy may be clinically indicated as first-line treatment in certain children. ${ }^{29}$ Palatine tonsil hypertrophy rarely causes adult OSA ${ }^{30}$ and thus isolated adenotonsillectomy does not yield much improvement in OSA in adults.

Adenotonsillectomy: Can consider as first-line for appropriate pediatric OSA patients but is rarely used as a sole treatment for adult OSA patients.

Nasal surgeries Nasal obstruction due to turbinate hypertrophy or septal deviation may compound snoring and OSA. ${ }^{31}$ Intranasal pathology increases upper airway resistance with subsequent collapse, which leads to hypopneas. ${ }^{31}$ Surgical techniques such as septoplasty with turbinate cautery and removal of concha bullosa can decrease airway resistance, which can treat OSA in some and can facilitate CPAP comfort for others. ${ }^{31}$

Nasal surgery: May cure some patients of their OSA; may allow others to try a smaller mask with a lower CPAP setting. 
Palatal surgeries Uvulopalatopharyngoplasty (UPPP) was developed to open a narrow or collapsed retropalatal area. Friedman et al. ${ }^{32}$ developed a staging system that incorporates palate position and BMI to estimate the rate of postsurgical success. Compared to the Mallampati classification, which assess patency of the oral airway with the tongue protruded, the Friedman tongue position is assessed with the tongue in a natural, neutral position. The essence of the Friedman staging system considers patients with a patent oral airway and BMI $<40 \mathrm{~kg} / \mathrm{m}^{2}$ as stage I; patients with a moderately crowded oral airway and BMI $<40 \mathrm{~kg} / \mathrm{m}^{2}$ as stage II; patients with either a very crowed oral airway (any BMI) or BMI $>40 \mathrm{~kg} / \mathrm{m}^{2}$ (any patency of oral airway) as stage III. Studies suggest that stages I, II, and III patients who underwent UPPP had respective success rates of $80.6 \%, 37.9 \%$, and $8.1 \% .^{32}$ Moderate to severe postoperative OSA ${ }^{25}$ still requires treatment, either with PAP therapy or an alternative such as an oral appliance.

Other palatal procedures include palatal z-plasty and pillar procedure. The pillar procedure reduces snoring by slightly stiffening the soft palate to mitigate its compliance and was found to lower AHI from $16.5 \pm 4.4 / \mathrm{h}$ to $11.2 \pm 10.2 / \mathrm{h}$ in patients with mild to moderate OSA. ${ }^{33}$ Palatal z-plasty, developed for post-tonsillectomy patients who continued to have air obstruction issues and were not candidates for an UPPP, ${ }^{34}$ has been shown to reduce snoring in patients with Friedman palate position II and III and may also lower the AHI. In a prospective study comparing UPPP and palatal z-plasty, in which surgical success was defined as a minimum $50 \%$ reduction in AHI and postoperative AHI less than $20 / \mathrm{h}$, surgical success occurred in $28 \%$ of UPPP patients with a preoperative AHI of $33.4 \pm 13.9^{34}$ and in $68 \%$ of those who underwent palatal z-plasty with a preoperative AHI of $41.8 \pm 26.4 .^{34}$

Palatal surgeries: Patients with more a patent oral airway, nonsevere OSA, and BMI $<40 \mathrm{~kg} / \mathrm{m}^{2}$ may have better surgical outcomes.

Tongue-based surgeries Genioglossus advancement with hyoid suspension for treatment of OSA met the surgical criteria for success (defined as a minimum 50\% reduction in AHI and a postoperative AHI less than 20/h) for $70 \%$ of the participants involved in the study. ${ }^{35}$ Genioglossus advancement with hyoid suspension is often an adjunctive intervention with UPPP. Outcomes involving multiple oral-pharyngeal surgical interventions with UPPP showed an overall success rate of $76 \% .^{32,36}$

Transoral robotic surgery, developed to address tongue base and supraglottic obstruction, has been performed as a stand-alone procedure or as part of a multilevel pharyngeal, palatal, or nasal surgery. ${ }^{37}$ One study was able to demonstrate $84.3 \%$ of patients to have improvement in their preoperative vs postoperative AHI ( 42.7 vs 22.2 , respectively). In this study, $51.2 \%$ of patients met the surgical criteria for success, and $14 \%$ met the criteria for cure as defined as AHI $<5 / \mathrm{h}^{38}$ Individuals with a BMI $<30 \mathrm{~kg} / \mathrm{m}^{2}$ had a $15.3 \%$ cure rate, compared to $11.1 \%$ cure rate in those with a BMI $>30 \mathrm{~kg} / \mathrm{m}^{2} .{ }^{38}$

Multilevel surgeries Multilevel surgeries for OSA address multiple areas of obstruction along the upper airway. These may include nasal, palatal, and tongue surgeries, ${ }^{39}$ while other articles have described only palatal and tongue-based surgeries. ${ }^{25}$ Multilevel surgeries can be performed simultaneously or in a phased sequence, which may include phase I and phase II procedures.

Tongue-based surgeries: May be performed in isolation or as part of multilevel surgery; patients with $B M I<30 \mathrm{~kg} / \mathrm{m}^{2}$ may have better surgical outcomes.

\section{What are the subsequent (phase II) surgeries to consider for my OSA patients?}

Maxillomandibular advancement (MMA) MMA enlarges the velo-orohypopharyngeal airway via advancement of anterior pharyngeal tissues attached to the mandible. ${ }^{22-24}$ This procedure is sometimes reserved for patients with major maxillomandibular deficiency. ${ }^{22}$ For the best outcome, surgical candidates should have a BMI of $\leq 30 \mathrm{~kg} / \mathrm{m}^{2}$, cephalometric posterior airway space $<9 \mathrm{~mm}$ defining hypopharyngeal narrowing, veloorohypopharyngeal narrowing, and potential retrognathia. ${ }^{40}$ Typically seen as a final approach in stepwise phase surgery, and deemed phase II, MMA alone has been shown 


\section{Patients with OSA and comorbid obesity should} be counselled on long-term weight

\section{management.}

to be as effective as UPPP and MMA combined. ${ }^{41}$ In a review of 234 individuals, $90 \%$ of whom were men with a mean BMI of $29.1 \mathrm{~kg} / \mathrm{m}^{2}$ and preoperative mean AHI of $54.4 / \mathrm{h}$, MMA led to $87 \%$ reduction in AHI of with a mean postoperative AHI of $7.7 / \mathrm{h}^{22-24}$ Postsurgical facial aesthetics is generally discussed with the patients prior to the procedure. The maxillomandibular complex is advanced between 10 and $12 \mathrm{~mm}$ routinely in OSA patients, causing excessive maxillomandibular protrusion, ${ }^{42}$ which sometimes results in an ape-like appearance.

Maxillomandibular advancement: Consider for patients with hypopharyngeal narrowing, veloorohypopharyngeal narrowing, and retrognathia; patients with $B M I<30 \mathrm{~kg} / \mathrm{m}^{2}$ may have better surgical outcomes.

\section{What surgical OSA treatment targets neuromuscular tone?}

Hypoglossal nerve stimulation This pacemaker-like device is connected to a wire that attaches a small cuff to the hypoglossal nerve. ${ }^{43,44}$ When turned on, the device stimulates the hypoglossal nerve and protrudes the tongue, opening the pharyngeal airway and maintaining patency. ${ }^{43,44}$ Hypoglossal nerve stimulation reduces the AHI by $68 \%$, from 29.3 events to 9 events per hour, ${ }^{43}$ with sustained benefit at 3-year follow-up. ${ }^{44}$ Participants with a BMI of $>32 \mathrm{~kg} / \mathrm{m}^{2}$ and those with concentric retropalatal collapse on drug-induced sleep endoscopy are not candidates for hypoglossal nerve stimulation. ${ }^{43}$

Hypoglossal nerve stimulation: Consider for patients with $B M I<32 \mathrm{~kg} / \mathrm{m}^{2}$; drug-induced sleep endoscopy is required to determine the patient's candidacy; concentric retropalatal collapse is a contraindication.

\section{What treatments exist for OSA patients who do not want any device or surgery?}

Weight loss Patients with OSA and comorbid obesity should be counseled on long-term weight management. A goal BMI $<25 \mathrm{~kg} / \mathrm{m}^{2}$ through dietary or surgical weight loss may improve the AHI in obese patients with OSA. ${ }^{45}$ Major weight loss in conjunction with PAP therapy may allow for reduced PAP requirements, ${ }^{45}$ which may facilitate patient comfort. Weight loss may also benefit management of other existing comorbidities such as hypertension and high cholesterol. ${ }^{45}$

Weight loss: Consider for all overweight or obese patients with OSA.

Positional therapy Positional therapy was developed to keep patients in a nonsupine position. Supine position can decrease the area of the upper airway and worsen severity of OSA. Avoidance of supine sleep, with isolated lateral positioning, could potentially increase airway patency. ${ }^{46}$ Patients who pursue positional therapy are advised to wear a device, either a commercially available product or homemade positioner, to prevent supine sleep.

Positional therapy: Consider avoidance of supine sleep for patients whose OSA is worse while back-sleeping.

Nasal expiratory PAP Nasal expiratory PAP is a Food and Drug Administration-cleared device that relies on patent nasal passages. A randomized controlled trial showed a $42.7 \%$ decrease in AHI with use of the device. ${ }^{47}$ The device valve rests in the nose and acts as a one-way resistor, permitting unobstructed inspiration. ${ }^{48}$ Airflow during expiration increases resistance, which creates expiratory PAP during expiration that is maintained until the start of the next inspiration. ${ }^{48}$ 
Nasal expiratory PAP: May consider for patients with patent nasal passages who do not mouth-breathe during sleep; less studied compared to other OSA treatments.

Noninvasive oral pressure therapy Oral pressure therapy is delivered via a device that rests within the mouth. The device produces a negative pressure with a vacuum within the oral cavity, which draws the soft palate forward and stabilizes the airway. ${ }^{49}$ Although one study suggested significant improvement in AHI with use of oral pressure therapy, this benefit was not seen among all groups of patients in the study. ${ }^{49}$

Noninvasive oral pressure therapy: May consider for patients who can tolerate the device; less studied compared to other OSA treatments.

\section{CONCLUSION}

Patients with neurologic disease who report fatigue and tiredness may have comorbid OSA. Diagnosis and treatment of OSA may contribute to improved quality of life for patients with neurologic disease. Continuous positive airway therapy continues to be the gold standard OSA treatment, and other modalities of PAP delivery are also available. Patients who cannot use or do not tolerate CPAP may require a multidisciplinary approach to care with collaboration among neurologists, sleep medicine specialists, dentists, and surgeons to determine the most appropriate management. This tailored approach can help identify an individual patient's specific sites of airway obstruction and develop a treatment plan that may maximize benefit to the patient.

\section{REFERENCES}

1. Basner R. Continuous positive airway pressure for obstructive sleep apnea. N Engl J Med 2007;356: 1751-1758.

2. Epstein LJ, Kristo D, Strollo PJ Jr, et al. Clinical guideline for the evaluation, management and longterm care of obstructive sleep apnea in adults. J Clin Sleep Med 2009;5:263-276.

3. Dyken ME, Im KB. Obstructive sleep apnea and stroke. Chest 2009;6:1668-1677.

4. Malow BA, Levy K, Maturen K, et al. Obstructive sleep apnea is common in medically refractory epilepsy patients. Neurology 2000;55:1002-1007.

5. Gander P, Scott G, Mihaere K, et al Societal costs of obstructive sleep apnoea syndrome. N Z Med J 2010;123:13-23.

6. Hillman DR, Murphy AS, Pezzullo L. The economic cost of sleep disorders. Sleep 2006;29:299-305.

7. Becker HF, Jerrentrup A, Ploch T. Effect of nasal continuous positive airway pressure treatment on blood pressure in patients with obstructive sleep apnea. Circulation 2003;107:68-73.

8. Giles T, Lasserson TJ, Smith BJ, et al. Continuous positive airways pressure for obstructive sleep apnea in adults. Cochrane Database Syst Rev 2006;3:CD001106.

9. Engleman HM, Wild MR. Improving CPAP use by patients with the sleep apnoea/hypopnoea syndrome (SAHS). Sleep Med Rev 2003;7:81-99.

10. Catcheside PG. Predictors of continuous positive airway pressure adherence. F1000 Med Rep 2010;2:70.

11. Morgenthaler TI, Aurora RN, Brown T, et al. Practice parameters for the use of autotitrating continuous positive airway pressure devices for titrating pressures and treating adult patients with obstructive sleep apnea syndrome: an update for 2007. Sleep 2008;31:141-147.

12. Selim BJ, Junna MR, Morganthaler TI. Therapy for sleep hypoventilation and central apnea syndromes. Curr Treat Options Neurol 2012;14:427-437.

13. Stell IM, Paul G, Lee KC, et al. Noninvasive ventilator triggering in chronic obstructive pulmonary disease. Am J Respir Crit Care Med 2001;164:2092-2097.

14. Radunovic A, Annane D, Rafiq MK, et al. Mechanical ventilation for amyotrophic lateral sclerosis/motor neuron disease. Cochrane Database Syst Rev 2013;3:CD004427.

15. Nolan GM, Doherty LS, Mc Nicholas WT. Auto-Adjusting versus fixed positive pressure therapy in mild to moderate obstructive sleep apnea. Sleep 2007;30:189-194. 
16. Gagnadoux F, Fleury B, Vielle B, et al. Titrated mandibular advancement versus positive airway pressure for sleep apnea. Eur Respir J 2009;34:914-920.

17. Haskell JA, McCrillis J, Haskell BS, et al. Effects of mandibular advancement device (MAD) on airway dimensions assessed with cone-beam computed tomography. Semin Orthod 2009;15:132-158.

18. Remmers J, Charkhandeh S, Grosse J, et al. Remotely controlled mandibular protrusion during sleep predicts therapeutic success with oral appliances in patients with obstructive sleep apnea. Sleep 2013; 36:1517-1525.

19. Ramar K, Dort LC, Katz SG, et al. Clinical practice guideline for treatment of obstructive sleep apnea and snoring with oral appliance therapy: an update for 2015. J Clin Sleep Med 2015;11:773-798.

20. Deane SA, Cistulli PA, Ng AT. Comparison of mandibular advancement splint and tongue stabilizing device in obstructive sleep apnea: a randomized controlled trial. Sleep 2009;32:648-653.

21. Ngiam J, Balasubramaniam R, Darendeliler MA, et al. Clinical guidelines for oral appliance therapy in the treatment of snoring and obstructive sleep apnoea. Aust Dent J 2013;58:408-419.

22. Aurora RN, Casey KR, Kristo D. Practice parameters for the surgical modifications of the upper airways for obstructive sleep apnea in adults. Sleep 2010;33:1396-1407.

23. Conradt R, Hochban W, Brandenburg U, et al. Long-term follow-up after surgical treatment of obstructive sleep apnoea by maxillomandibular advancement. Eur Respir J 1997;10:123-128.

24. Giarda M, Brucoli M, Arcuri F, et al. Efficacy and safety of maxillomandibular advancement in treatment of obstructive sleep apnoea syndrome. Acta Otorhinolaryngol Ital 2013;33:43-46.

25. Caples SM, Rowley JA, Prinsell JR, et al. Surgical modifications of the upper airway for obstructive sleep apnea in adults: a systemic review and meta-analysis. Sleep 2010;33:1396-1407.

26. Won CH, Li KK, Guilleminault C. Surgical treatment of obstructive sleep apnea: upper airway and maxillomandibular surgery. Proc Am Thorac Soc 2008;5:193-199.

27. Hobson JC, Robinson S, Antic NA, et al. What is "success" following surgery for obstructive sleep apnea? The effect of different polysomnographic scoring systems. Laryngoscope 2012;122:1878-1881.

28. Kothare SV, Rosen CL, Lloyd RM, et al. Quality measures for the care of pediatric patients with obstructive sleep apnea. J Clin Sleep Med 2015;11:385-404.

29. Bhattancharjee R, Kheirandish-Gozal L, Spruyt K, et al. Adenotonsillectomy outcomes in treatment of obstructive sleep apnea in children: a multicenter restrospective study. Am J Respir Crit Care Med 2010;182:676-683.

30. Verse T, Kroker BA, Pirsig W, et al. Tonsillectomy as a treatment of obstructive sleep apnea in adults with tonsillar hypertrophy. Laryngoscope 2000;9:1556-1559.

31. Sufioglu M, Ozmen OA, Kasapoglu F, et al. The efficacy of nasal surgery in obstructive sleep apnea syndrome: a prospective clinical study. Eur Arch Otorhinolaryngol 2012;269:486-494.

32. Friedman M, Ibrahim HZ, Bass L. Clinical staging for sleep-disordered breathing. Otolaryngol Head Neck Surg 2002;127;13-21.

33. Goessler UR, Hein G, Verse T, et al. Soft palate implants as a minimally invasive treatment for mild to moderate obstructive sleep apnea. Acta Otolaryngol 2007;127:527-531.

34. Friedman M, Ibrahim HZ, Vidyasagar R, et al. Z-palatoplasty (ZPP): a technique for patients without tonsils. Otolaryngol Head Neck Surg 2004;131:89-100.

35. Neruntarat C. Genioglossus advancement and hyoid myotomy under local anesthesia. Otolaryngol Head Neck Surg 2003;129:85-91.

36. Jacobowitz O. Palatal and tongue base surgery for surgical treatment of obstructive sleep apnea: a prospective study. Otolaryngol Head Neck Surg 2006;135;258-264.

37. Thaler ER, Rassekh CH, Lee JM, et al. Outcomes for multilevel surgery for sleep apnea: obstructive sleep apnea, transoral robotic surgery, and uvulopalatopharyngoplasty. Laryngoscope 2016;126:266269.

38. Hoff P, Glazer TA, Spector ME. Body mass index predicts success in patients undergoing transoral robotic surgery for obstructive sleep apnea. J Otorhinolaryngol Relat Spec 2014;75:266-272.

39. Pang KP, Siow JK, Tseng P. Safety of multilevel surgery in obstructive sleep apnea: a review of 487 cases. Arch Otolaryngol Head Neck Surg 2012;138:353-357.

40. Prinsell JR. Primary and secondary telegnathic maxillomandibular advancement, with or without adjunctive procedures, for obstructive sleep apnea in adults: a literature review and treatment recommendations. J Oral Maxillofac Surg 2012;70:1659-1677.

41. Boyd SB, Walters AS, Song Y. Comparative effectiveness of maxillomandibular advancement and uvulopalatopharyngoplasty for the treatment of moderate to severe obstructive sleep apnea. J Oral Maxillofac Surg 2013;71:743-751.

42. Li KK, Riley RW, Powell NB, et al. Patient's perception of the facial appearance after maxillomandibular advancement for obstructive sleep apnea syndrome. J Oral Maxillofac Surg 2001;59:377-380.

43. Strollo PJ Jr, Soose RJ, Maurer JT, et al. Upper-airway stimulation for obstructive sleep apnea. N Engl J Med 2014;370;139-149. 
44. Woodson BT, Soose RJ, Gillespie MB, et al. Three-year outcomes of cranial nerve stimulation for obstructive sleep apnea: the STAR Trial. Otolaryngol Head Neck Surg 2016;154:181-188.

45. Morgenthaler TI, Kapen S, Lee-Chiong T, et al. Practice parameters for the medical therapy of obstructive sleep apnea. Sleep 2009;29:1031-1035.

46. Pevernagie DA, Stanson AW, Sheedy PF II, et al. Effects of body position on the upper airway of patients with obstructive sleep apnea. Am J Resp Crit Care Med 1995;152;179-185.

47. Berry RB. A novel nasal expiratory positive airway pressure (EPAP) device for the treatment of obstructive sleep apnea: a randomized controlled trial. Sleep 2011;34:479-485.

48. Colrain IM, Black J, Siegel LC, et al. A multicenter evaluation of oral pressure therapy for the treatment. Sleep Med 2013;14:830-837.

49. Doshi R, Westbrook P. A novel non-prescription nasal EPAP device (Theravent) to treat snoring. Sleep Diagn Ther 2012;7:1-5.

Received April 27, 2016. Accepted in final form September 26, 2016.

\section{AUTHOR CONTRIBUTIONS}

Patrick Pavwoski: writing and critical revision of manuscript for intellectual content. Anita Valanju Shelgikar: writing and critical revision of manuscript for intellectual content.

\section{STUDY FUNDING}

No targeted funding reported.

\section{DISCLOSURES}

P. Pavwoski reports no disclosures. A.V. Shelgikar performs clinical procedures or imaging studies related to sleep medicine (65\% effort). Full disclosure form information provided by the authors is available with the full text of this article at Neurology.org/cp.

\section{Related articles from AAN physician and patient resources}

\section{Neurology ${ }^{\circledR} \quad$ Neurology.org}

Role of sleep-disordered breathing and sleep-wake disturbances for stroke and stroke recovery September 27, 2016;87:1407-1416.

Teaching NeuroImages: Obstructive sleep apnea triggered by vagus nerve stimulation November 3, 2015;85:e140.

\section{Continuum ${ }^{\circledR}$ - ContinuumJournal.com}

Risk Factor Management for Stroke Prevention April 2014;20:296-308.

\section{Neurology Now ${ }^{\circledR}$ - Neurologynow.com}

Sleep Smarter: For people with neurologic conditions, sleep can be elusive. We troubleshoot six obstacles to a healthy night's rest

April/May 2016:12:44-51.

Quick Tips: Sleep Wise: Avoid the health risks of sleep apnea with this expert advice April/May 2015;11:7.

\section{Neurology Today ${ }^{\circledR} \quad$ - Neurotodayonline.com}

NEWS FROM THE INTERNATIONAL STROKE CONFERENCE: Sleep Apnea Linked to Brainstem Infarcts

March 20, 2014;14:14. 